\title{
UPPER AND LOWER FIELDS FOR PROFINITE GROUPS OF GIVEN CARDINALITY
}

\author{
DON KRAKOWSKI
}

(Received I October 1972)

Communicated by J. P. O. Silberstein

Profinite groups (that is, compact totally disconnected topological groups) have been characterized as Galois groups in Krakowski (1971) and Leptin (1955). They can, in fact, be realized as groups of permutations on sets of transcendentals where every transcendental has a finite orbit under the group action and the fixed field is generated by the invariant rational functions on these orbits. If $G=$ $\left\{G_{+\alpha} \mid \alpha \in A\right\}$ we define the cardinality of $G$ as $|G|=|A|$. In this paper we construct, for every cardinal number $c$ and profinite group $G$ with $|G| \leqq c$ two universal fields $k_{c}$ and $K_{c}$ so that $G \simeq \operatorname{Gal}\left(K_{c} / K_{G}\right)$ and $G \simeq\left(K_{G} / k_{c}\right)$ for fields $K_{G}$ and $k_{G}$ which depend on $G$. See Cassels and Frohlich (1967) for a description of the basic properties of profinite groups.

THEORFM 1. Let $p$ be sero or a prime number, and c any cardinal number. Then there is a field $K_{c}$ of characteristic $p$ such that for every profinite group $G$ with $|G| \leqq c$ there exists a subfield $K_{G}$ over which $K_{c}$ is Galois, and $\operatorname{Gal}\left(K_{c} / K_{G}\right) \cong G$.

PROOF. Let $L$ be a field of characteristic $p$, and $I$ be an index set such that $|I|=c$. Define

$$
K_{c}=L\left(\left\{X_{\text {in }} \mid i \in I, n \in Z^{+}\right\}\right),
$$

$K_{i j}$ are independent indeterminates over $L$.

If $G=\left\{G_{-\alpha} \mid \alpha \in A\right\}$, with $|A| \leqq c$, regard $A$ as embedded in $I$ and $G_{\alpha}$ as emuedded in $S_{n_{x}}$, the symmetric group, where $n_{\alpha}=\left|G_{\alpha}\right|$.

If $\sigma \in G$ we define

$$
\sigma\left(X_{\beta j}\right)= \begin{cases}X_{\alpha \sigma(j)} & \text { if } \alpha=\beta \text { and } j \leqq n_{\alpha} \\ X_{\beta j} & \text { if } \alpha \neq \beta \text { or } \alpha=\beta \text { and } j>n_{\alpha} .\end{cases}
$$

Let $\left.k=L\left(\left(\bigcup_{\alpha \in A} P\right) \cup\left(\bigcup_{\alpha \in A} X_{\alpha j} \mid j>n_{\alpha}\right\}\right)\right)$, where $P_{\alpha}$ is the set of polynomials in $\left\{X_{\alpha 1}, \cdots, X_{\alpha n_{x}}\right\}$ invariant under $G_{\alpha} . K_{c}$ is a union of Galois extensions over $k$ 
and hence is itself Galois over $k$. If we show that $\mathrm{Gal}\left(K_{c} / k\right)=\prod_{\alpha \in A} G_{\alpha}$ then, since $G=G_{\leftarrow \alpha}$ is closed in $\Pi_{\alpha \in A} G_{\alpha}$ [Cassels and Frohlich (1967)] it follows from the fundamental theorem of the infinite Galois theory Jacobson (1964) that there is an intermediate field $k \subseteq K_{G} \subseteq K_{c}$ and $G \cong \mathrm{Gal}\left(K_{c} / K_{G}\right)$. To show Gal $\left(K_{c} / k\right)=\Pi_{\alpha \in A} G_{\alpha}$ we define

$$
\left.k_{\alpha}=k\left(\left\{X_{\alpha 1}, \cdots, X_{\alpha n_{\alpha}}\right\}\right) \cup\left(\bigcup\left\{X_{\beta j} \mid \beta \neq \alpha\right\}\right)\right) .
$$

Then, as above, the action of $G_{\alpha}$ is on subscripts and it is evident that $k_{\alpha}$ is Galois over $k$, with $\operatorname{Gal}\left(k_{\alpha} / k\right) \cong G_{\alpha}$. Let $K_{\alpha}=\bigcup_{\beta \leqq \alpha} k_{\beta}$. Since $k_{\alpha} \cap k_{\beta}=k$ for $\alpha \neq \beta$, and $\Pi_{\beta \leqq \alpha} k_{\beta}$ (the composition of the $\left.k_{\beta}{ }^{\prime} s\right)=K_{\alpha}$, we conclude that $\operatorname{Gal}\left(K_{\alpha} / k\right)$ $\simeq \Pi_{\alpha \leqq \beta} G_{\alpha}$. Noting that $\bigcup_{\alpha \in A} K_{\alpha}=K_{c}$, we obtain $\operatorname{Gal}\left(K_{c} / K\right) \simeq \Pi_{\alpha \in A} G_{\alpha}$ and our conclusion follows.

THEOREM 2. Let $p$ be a zero or a prime number, c any cardinal number. Then there is a field $k_{c}$ of characteristic $p$ such that for every profinite group $G$ with $|G| \leqq c$ there exists a Galois extension $k_{G}$ of $k_{c}$ with $\operatorname{Gal}\left(k_{G} / k_{c}\right) \simeq G$.

Proof. Let $\Pi$ be the set of profinite groups $G$ with $|G| \leqq c$, where we take one per isomorphism class. We first show that, without loss of generality, $|G|=c$ for every $G \in \Pi$. If $G=\left\{G_{\leftarrow-\alpha} \mid \alpha \in A\right\}$, let $I$ be a set with $|I|=c$ and consider $A$ as embedded in $I$. Pick $\alpha \in A$ and let $\leqq$ be the quasi order on $A$ (as a directed set). For every $i \in I, i \notin A$ let $\alpha_{i}$ be a symbol and define the set $A^{\prime}=A \cup\left\{\alpha_{i} \mid i \in I\right.$, $i \notin A\}$. Extend the quasi order on $A$ to $A^{\prime}$ by defining for each pair $i, j$ in $I \backslash A$, and each $a \in A$ :

$$
\begin{aligned}
& \alpha_{i} \leqq \alpha_{j} \\
& \alpha_{i} \leqq a \text { if and only if } \alpha \leqq a \\
& a \leqq \alpha_{i} \text { if and only if } a \leqq \alpha .
\end{aligned}
$$

This makes $A^{\prime}$ into a directed set, $\left|A^{\prime}\right|=c$. Now take the profinite group

$$
G^{\prime}=\left\{G_{+\beta}^{\prime} \mid \beta \in A^{\prime} ; G_{\beta}^{\prime}=G_{\beta} \text { if } \beta \in A, G_{\beta}^{\prime}=G_{\alpha} \text { if } \beta=\alpha_{i}\right\},
$$

with defining homomorphism on $\pi_{\alpha_{i}}^{\beta}=\pi_{x}^{\beta}, \pi_{\beta}^{\alpha_{i}}=\pi_{\beta}^{\alpha}, \pi_{\alpha_{j}}^{\alpha_{i}}=$ identity. Since $A$ is cofinal in $A^{\prime}$, we have $G \simeq G^{\prime}$.

Now let $G_{\mu}=\left\{G_{+}{ }^{(\mu)} \mid i \in I\right\} \in \Pi$, and embed $G_{i}^{(\mu)}$ in the symmetric group $S_{\mu i}^{(\mu)}$, where $\left|G_{i}^{(\mu)}\right|=n_{i}^{(\mu)}$. We introduce the set of independent indeterminates

$$
\left\{X_{\mu i n} \mid \mu \in \Pi, i \in I, n \in Z^{+}\right\}
$$

upon which $G_{\mu}$ acts as follows: for $\sigma \in G_{\mu}$ with $\sigma \in G_{i}^{(\mu)}$

$$
\sigma\left(X_{\mathrm{vi}_{\mu}}\right)= \begin{cases}X_{\mu i \sigma_{i}(n)} & \text { if } v=\mu \\ X_{\text {in }} & \text { if } v \neq \mu .\end{cases}
$$


Let $L$ be an arbitrary field of characteristic $p$, and define $k_{c}=L\left(\bigcup_{\mu \in \infty} P_{\mu}\right)$ where $P_{\mu}$ is the set of polynomials in $X_{\mu \text { in }}\left(i \in I, n \in Z^{+}\right)$invariant under $G_{\mu}$. Let $k_{\mu}=k_{c}\left(\left\{X_{\mu i n} \mid i \in I, n \in Z^{+}\right\}\right)$. Then $k_{\mu}$ is Galois over $k_{c}$ with Galois group $G_{\mu}$.

RemarK. The mere existence of the fields $K_{c}$ and $k_{c}$ can be demonstrated by a more simple argument: Let $c$ be the given cardinal and form the product $P=\Pi G_{\alpha}$, where the product is extended over all profinite groups $G_{\alpha}$, with $\left|G_{\alpha}\right| \leqq c$, and we choose one group per isomorphism class. Since the product of profinite groups is again profinite, $P$ is profinite and hence a Galois group, Krakowski (1971). Thus, there exist fields $K_{c}$ and $k_{c}$, of any prescribed characteristic, such that $P=\operatorname{Gal}\left(K_{c} / k_{c}\right)$. Then by the fundamental theorem of the infinite Galois theory, for every profinite group $G$ with $|G| \leqq c$ there exist fields $K_{1}$ and $K_{2}$ with $k_{c} \subseteq K_{i} \subseteq K_{c}$ such that $\operatorname{Gal}\left(K_{1} / k_{c}\right) \simeq \operatorname{Gal}\left(K_{c} / K_{2}\right) \simeq G$. The existence of both an upper and lower field follows from the fact that $G$ is both a closed subgroup as well as a homomorphic image of $\boldsymbol{P}$.

\section{Acknowledgment}

This paper is based on some results in my thesis, done under Professor E. G. Strauss, to whom I wish to express my warm thanks.

\section{References}

J. W. S. Cassels and A. Frohlich (1967), Algebraic Number Theory, Chapter V; (Thompson Book Co., Washington D. C. (1967)).

N. Jacobson (1964), Lectures in Abstract Algebra. Vol. III, 1964, (D. Van Nostrand Co., Princeton, New Jersey, 1964).

D. Krakowski, Profinite Groups and the Galois Groups of Fields, (Thesis), (submitted to Univ. of Cal. at Los Angeles, June 1971).

H. Leptin (1955), 'Compact, totally disconnected groups', Arch. Math. 6, 371-373.

The Weizmann Institute of Science

Rehovot Israel.

Present address:

Department of Mathematics

Wayne State University

Detroit, Michigan 48202

U.S.A. 